\title{
Recurrent infection-induced autoimmune haemolytic anaemia complicated by pulmonary embolism: a case report and literature review
}

\author{
Authors: Shu Fang ${ }^{A}$ and Fan Yang ${ }^{B}$
}

\begin{abstract}
A 73-year-old woman presented with progressive dyspnoea up to type 1 respiratory failure. Laboratory values showed leucocytosis, reduced haemoglobin to $71 \mathrm{~g} / \mathrm{L}$, elevated indirect serum bilirubin and lactic dehydrogenase. Computed tomography pulmonary angiography (CTPA) revealed peripheral pulmonary embolism (PE). Echocardiography showed enlarged right ventricle, elevated estimated pulmonary arterial systolic pressure $(57.2 \mathrm{mmHg})$ and normal left ventricular ejection fraction. The patient was diagnosed with autoimmune haemolytic anaemia (AIHA), which was induced by recurrent infections without standard treatment in the past year. AIHA is the cause of PE due to the absence of common predisposing factors and other thrombophilia. The patient became better after administration of glucocorticoids, intravenous immunoglobulin and rivaroxaban.
\end{abstract}

KEYWORDS: autoimmune haemolytic anaemia, pulmonary embolism, infection

DOI: $10.7861 /$ clinmed.2021-0112

\section{Case presentation}

A 73-year-old woman was referred to the emergency department (ED) with progressive dyspnoea, weakness and dark urine for about 10 days. She complained of recurrent fever for 1 year due to pneumonia or urinary tract infection (UTI). She presented to local hospital and was diagnosed with suspicious infection due to leucocytosis. Although treating with moxifloxacin, her symptoms failed to relieve. Past medical history included reflux oesophagitis for many years and was treated with omeprazole. She has no history of illicit substance use.

Vital signs were blood pressure at $116 / 62 \mathrm{mmHg}$, heart rate of 90 beats/min, respiratory rate of 26 breaths/min and oxygen saturation of $85 \%$ on room air. Physical examination showed highpitched $\mathrm{P} 2$, no crackles and rhonchi in both lungs, no peripheral oedema, no erythema and no palpable cords. The initial significant

Authors: Aresident physician, Peking University First Hospital, Beijing, China; ${ }^{B}$ attending physician, Peking University First Hospital, Beijing, China findings revealed type 1 respiratory failure, haemoglobin $(\mathrm{Hb})$ was $71 \mathrm{~g} / \mathrm{L}$ with white cell count of $23.01 \times 10^{9} / \mathrm{L}$, neutrophils of $74.2 \%$ and platelet count of $241 \times 10^{9} / \mathrm{L}$; a high level of D-dimer ( $2.78 \mathrm{mg} / \mathrm{L}$ ); troponin I (CTNI) was $0.091 \mathrm{ng} / \mathrm{mL}$ and brain natriuretic peptide (BNP) was $236 \mathrm{pg} / \mathrm{mL}$. A 12-lead electrocardiography (ECG) showed no abnormalities in ST segment and T wave (Fig 1). Emergent computed tomography pulmonary angiography (CTPA) showed bilateral subsegmental and segmental pulmonary emboli (Fig 2a).

\section{Initial management}

The patient was sent to the cardiac care unit (CCU).

Echocardiography showed enlarged right ventricle and elevated estimated pulmonary arterial systolic pressure $(57.2 \mathrm{mmHg})$ and normal left ventricular ejection fraction (81.2\%). Dynamic changes of laboratory results are summarised in supplementary material S1. We exclude common predisposing factors and other thrombophilia for pulmonary embolism (PE) by a detailed history and laboratory test (cardiolipin anti-antibody, anti- $\beta 2$ glycoprotein 1 antibody, lupus anticoagulant, protein $\mathrm{C}$ or $\mathrm{S}$ were normal). The patient suffered from haemolytic anaemia as judged by elevated reticulocyte and serum bilirubin. Further tests showed peripheral blood smears, paroxysmal nocturnal haemoglobinuria (PNH) clone, haemoglobin electrophoresis, erythrocyte penetration fragility test, G6PD activity, complement $\mathrm{H}$ factor concentration, complement $\mathrm{H}$ factor antibody and ADAMTS13 were all normal. Coombs test was positive, and resulted in the diagnosis of autoimmune haemolytic anaemia

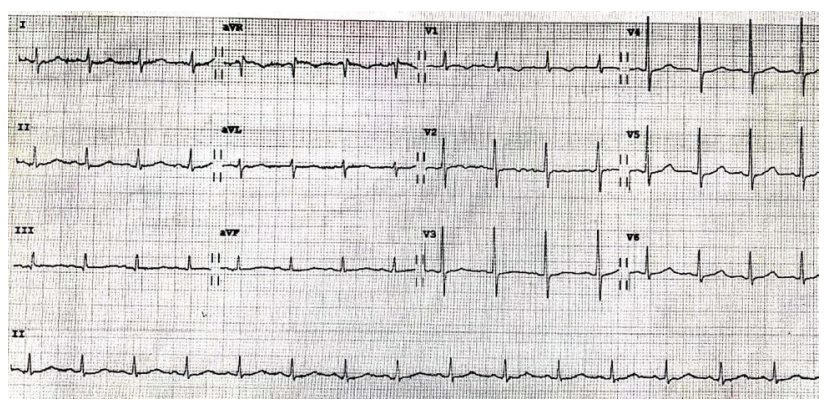

Fig 1. Electrocardiography on admission. 

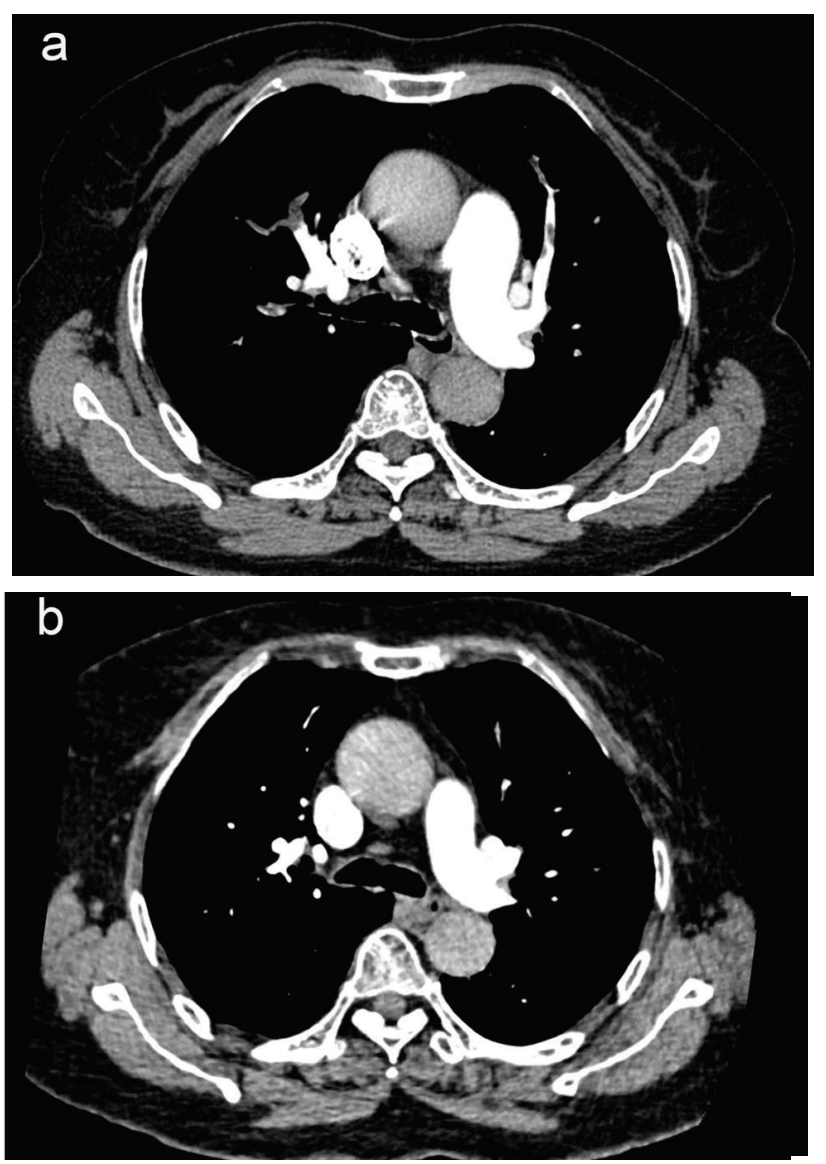

Fig 2. Comparison of computed tomography pulmonary angiography. a) Image on admission showing bilateral subsegmental and segmental pulmonary embolism. b) Image at 3 months after discharge showing no pulmonary embolism.

(AIHA). The works for secondary AIHA included other autoimmune diseases (antinuclear antibody, anti-dsDNA were negative), serum immunofixation electrophoresis (no monoclonal band) and a computed tomography to exclude lymphoma and solid tumours. In addition, T-SPOT.TB, the immunoglobulin M of mycoplasma, Epstein-Barr virus (EBV), cytomegalovirus (CMV), influenza virus, rubella virus, measles, adenovirus and parvovirus B19, hepatitis B surface antigen (HBsAg) and HIV antibodies were all negative. Finally, PE attributed to AIHA was considered due to no common aetiology of PE.

According to guidelines, the patient was stratified as Pulmonary Embolism Severity Index (PESI) class IV, simplified PESI (sPESI) $\geq 1$, the risk of early death is intermediate-high. ${ }^{1}$ She received low-molecular weight heparin (LMWH) and discontinued other suspicious drugs. During hospitalisation, she once received intravenous methylprednisolone sodium succinate 40 mg once daily (od)) and intravenous immunoglobulin $10 \mathrm{~g}$ od for 7 days. The patient didn't receive anti-infective nor blood transfusion therapy.

\section{Case progression and outcome}

The patient was discharged after 3 weeks with oral prednisolone $40 \mathrm{mg}$ qd and rivaroxaban $15 \mathrm{mg}$ twice daily (bid). At 3-month follow up, reinspection of CTPA revealed disappearance of thrombosis (Fig 2b). At the recent follow-up, 12 months after the initial presentation, she required $5 \mathrm{mg}$ od of prednisolone and $20 \mathrm{mg}$ od of rivaroxaban to keep her haemoglobin $(\mathrm{Hb})$ over $10 \mathrm{~g} / \mathrm{dL}$ and had no thromboembolic episodes.

\section{Discussion}

Autoimmune haemolytic anaemia (AIHA) is a rare and heterogeneous disorders characterised by the destruction of red blood cells through warm or cold autoantibodies. ${ }^{2}$ The overall estimated incidence in adults is $0.8-3$ per $100,000 /$ year, a prevalence of $17: 100,000$ and a mortality rate of $3-4 \%{ }^{3,4}$ AIHA is the higher risk for thromboembolism due to hypercoagulability. ${ }^{2}$ AIHA occur in systemic lupus erythematosus (SLE), chronic lymphocytic leukaemia (CLL), infection, drug exposure and other mixed disorders. ${ }^{2,5,6}$ As early as 1967, Allgood et al reported that pulmonary embolism (PE) was the most common cause of death in AIHA, and revealed the association between PE and AIHA. ${ }^{7}$ Patients with AIHA have 2.6-fold higher risk of venous thromboembolism (VTE) compared with non-AIHA. ${ }^{8}$ In two large studies, the prevalence of thromboembolism in AIHA patients was recorded up to $15 \% .4 .9$ Anticoagulant prophylaxis can effectively reduce the occurrence of a thromboembolic episode in acute exacerbation of AIHA ( $4.7 \%$ vs $33.3 \%) .{ }^{10}$ The reasons for patients with AIHA were at an increased risk of VTE remain unclear, which may include the increased expression of phosphatidylserine, microparticles and cytokine-induced monocytes or endothelial tissue factors. ${ }^{8,11}$ Thromboembolic episodes are relatively common during active phases of the disease, but also occur during disease maintenance therapy. ${ }^{12}$ High leucocyte count and severe anaemia were associated with VTE. ${ }^{9}$ PE is a life-threatening complication of AIHA. Clinical manifestations of PE include sudden dyspnoea, hypoxaemia, and chest pain, which can easily be masked by severe anaemia caused by AIHA, leading to delayed diagnosis. ${ }^{13}$ Therefore, close attention as well as timely treatment plays a crucial role.

The causes of AIHA and PE are complex and varied, which need comprehensive evaluation in patients with $\mathrm{PE}$ at the first occurrence. ${ }^{1,2}$ In our case, considering that the patient had suffered recurrent infection without sufficiently effective treatment and haemoglobin gradually decreased in the past year, which strongly suggested that the prodromal infection induced AIHA. Previous literature reported that infection associated AIHA is not rare, accounting for $14 \%$ of all patients. ${ }^{11}$ Drug-related AIHA was excluded because the anaemia did not worsen after taking drugs such as moxifloxacin.

The first-line therapy for AIHA is glucocorticoids and approximately $80 \%$ of patients would respond to the therapy. There are no studies in comparing tapering regimens, where long duration and slow reduction are recommended. No response to steroids after 21 days or relapse during/after a steroid wean is an indication for the second-line therapy. ${ }^{2,3}$ This patient was administered with rivaroxaban sequent to $L M W H$, although there was insufficient evidence-based practice in the alternative of novel oral anticoagulants to vitamin K antagonists. Direct oral anticoagulants (DOACs) are not recommended in antiphospholipid syndrome (APS)-induced VTE.14 Notably, numbers of AIHA patients complicated with thromboembolism were reported with positive antiphospholipid antibodies. ${ }^{11}$ The thrombotic risk is reported to not correlate with traditional risk factors during AIHA. ${ }^{13}$ 
Vitamin $\mathrm{K}$ antagonists reduce functional coagulation factors in the extrinsic and intrinsic pathways of coagulation, which showed a stronger anticoagulant effect. ${ }^{15}$ Thus, it is uncertain that DOACs may be a perfect substitute to warfarin in VTE, especially attributed to thrombophilia or autoimmune diseases. In our case, DOACs are safe and effective for this patient, which providing a clinical evidence for anticoagulant therapy of AIHA-induced PE. Given the higher risk of thrombosis, long-term anticoagulation and follow-up should be recommended. ${ }^{12}$

\section{Key points}

> AIHA is a hypercoagulable disorder, requiring vigilance against the complication of deep vein thrombosis.

$>$ Consider a wide range of differentials when patients present with PE attributed to AIHA.

$>$ No guidelines to recommend anticoagulant therapy for AIHA induced PE. DOACs may be effective and safe in APS-negative AIHA. .

\section{Supplementary material}

Additional supplementary material may be found in the online version of this article at www.rcpjournals.org/clinmedicine: S1 - Dynamic changes of laboratory results and process of diagnosis and treatment from the initial infection to follow-up.

\section{Acknowledgements}

Written informed consent was obtained from the patient to publish the clinical details and images in this article.

\section{References}

1 Konstantinides SV, Meyer G, Becattini C et al. 2019 ESC Guidelines for the diagnosis and management of acute pulmonary embolism developed in collaboration with the European Respiratory Society (ERS). Eur Heart J 2020;41:543-603.

2 Jäger $\mathrm{U}$, Barcellini W, Broome CM et al. Diagnosis and treatment of autoimmune hemolytic anemia in adults: Recommendations from the First International Consensus Meeting. Blood Rev 2020; 41:100648

3 Hill QA, Stamps R, Massey E et al. The diagnosis and management of primary autoimmune haemolytic anaemia. $\mathrm{Br}$ ] Haematol 2017:176:395-411.
4 Barcellini W, Zaninoni A, Fattizzo B et al. Predictors of refractoriness to therapy and healthcare resource utilization in 378 patients with primary autoimmune hemolytic anemia from eight Italian reference centers. Am J Hematol 2018;93:e243-6.

5 Newman K, Owlia MB, El-Hemaidi I, Akhtari M. Management of immune cytopenias in patients with systemic lupus erythematosus - Old and new. Autoimmunity Reviews 2013;12:784-91.

6 Hodgson K, Ferrer G, Pereira A, Moreno C, Montserrat E. Autoimmune cytopenia in chronic lymphocytic leukaemia: diagnosis and treatment. Br J Haematol 2011;154:14-22.

7 Allgood JW, Chaplin H, Jr. Idiopathic acquired autoimmune hemoIytic anemia. A review of forty-seven cases treated from 1955 through 1965. Am J Med 1967;43:254-73.

8 Ungprasert P, Tanratana P, Srivali N. Autoimmune hemolytic anemia and venous thromboembolism: A systematic review and metaanalysis. Thromb Res 2015;136:1013-7.

9 Barcellini W, Fattizzo B, Zaninoni A et al. Clinical heterogeneity and predictors of outcome in primary autoimmune hemolytic anemia: a GIMEMA study of 308 patients. Blood 2014;124:2930-6.

10 Hendrick AM. Auto-immune haemolytic anaemia--a high-risk disorder for thromboembolism? Hematology 2003;8:53-6.

11 Hoffman PC. Immune hemolytic anemia--selected topics. Hematology Am Soc Hematol Educ Program 2009:80-6.

12 Audia S, Bach B, Samson M et al. Venous thromboembolic events during warm autoimmune hemolytic anemia. PLoS One 2018;13:e0207218.

13 Lecouffe-Desprets M, Néel A, Graveleau ] et al. Venous thromboembolism related to warm autoimmune hemolytic anemia: a case-control study. Autoimmunity Reviews 2015;14:1023-8.

14 Pengo V, Denas G, Zoppellaro G et al. Rivaroxaban vs warfarin in high-risk patients with antiphospholipid syndrome. Blood 2018;132:1365-71.

15 Cohen $\mathrm{H}$, Hunt B], Efthymiou M et al. Rivaroxaban versus warfarin to treat patients with thrombotic antiphospholipid syndrome, with or without systemic lupus erythematosus (RAPS): a randomised, controlled, open-label, phase 2/3, non-inferiority trial. Lancet Haematology 2016;3:e426-36.

Address for correspondence: Dr Fan Yang, Department of Cardiology, Peking University First Hospital, No.8 Xishiku Street, Xicheng District, Beijing 100034, China. Email: dryangf@126.com 